\title{
Prototype for Wireless Charger based on Rectenna and Atmel Microcontroller
}

\author{
M. Swarnalatha ${ }^{1}$, K. Arthi ${ }^{2}$, T.R. Sree Vidya ${ }^{3}$ \\ Student, ECE, Valliammai College of Engineering, Chennai, India ${ }^{1,3}$ \\ Assistant Professor, ECE, Valliammai College of Engineering, Chennai, India ${ }^{2}$
}

\begin{abstract}
The amazing advancement of the cell phone has really refreshed the minds of the people, it helps for communication, multitask, transactions etc. Battery life of cellular phone is dependably been an issue for producers. Individuals are grumbling about their versatile battery life, that they don't have long battery life and they need to charge their phones. Cell phone charger can serve an important purpose to cell phone because it is used as the source of emergency power. Recharging of the battery in mobile phone seems to be complicated task. In this paper we would like to explain the prototype for charging the mobile phone using microwave wireless communication. It is the way of charging a device using microwave signals which can eliminate the use of cables, power adapter, etc. The prototype is a first or preliminary version of a device from which other forms are developed. The microwave signal is transmitted from transmitter along with message signal using slotted waveguide antenna at frequency $2.45 \mathrm{ghz}$. The charging is done by receiving signal with help of rectenna in phone then passes to microwave power transfer system(MPT). A rectenna is rectifying antenna that is used for converting electromagnetic energy to direct current (DC). Microwave power transfer (MPT) system transfer dc current into mobile phone as charge signal by using ATMEL microcontroller. Charging is done automatically, when call connected in your mobile phone and has we talk in the mobile phone.
\end{abstract}

Keywords: Rectenna, Wireless Charging, Microwave Power Transfer, Atmel Microcontroller.

\section{INTRODUCTION}

Radio spectrum covers radio waves and microwaves. Optical spectrum covers infrared ,visible,ultraviolet, $\mathrm{x}$ rays and gamma radiation. Radio waves ranges from $3 \mathrm{khz}$ to $300 \mathrm{Ghz}$ while microwaves from $300 \mathrm{Mhz}$ to $300 \mathrm{Ghz}$. Hence RF starts from much lower than microwave starting range .In radio waves, antenna wavelength varies from 100 meter to about 1 millimeter. The mobile phone used RF frequency for its operation,we choose $\mathrm{s}$ band of microwave region ( 2 to $4 \mathrm{ghz}$ ).Select license free 2.45ghz Industrial, Scientific and Medical(ISM) Radio band.A cell phone is essentially a two way radio consisting of a radio transmitter and a radio receiver. Cell phones are use radio waves to communicate. Radio waves transport digitized voice or data in form of oscillating electric and magnetic fields called the electro magneticfield(emf). The rate of oscillation is called frequency. Radio waves carry the information and travel in air at the speed of light. Cell phones transmit radio waves in all the direction. The waves can be absorbed and reflected by surrounding object before they reach the nearest cell tower. An antenna is an metallic element engineered to be a specific size and shape for transmitting and receiving specific frequency of radio waves, it is important to understand the any metallic component in the device can be interact with transmission antenna and contribute to the pattern of the transmitted signal. The microwave power transfer (MPT) system works by converting power to microwave through a microwave generator and then transmitting the power through free space where it is received and converting back to power by a special device called a rectenna. When the call is being connected through base station the usage microwave signal for communication takesplace remaining high frequency signal is used for charging purpose. Rectenna starts converting microwaves to dc power. Then the converted dc power acts as an input to analog to digital convertor(ADC). The ATMEL microcontroller reads the digital data send by the ADC then converts into output voltage and display in LCD about charging. Output is transferred through USB to mobile phones.

\section{TRANSMITTER SECTION}

The microwave signal is transmitted from transmitter along with message signal using slotted wave guides antenna at frequency $2.45 \mathrm{ghz}$. A waveguide is a structure that the guides waves such as electromagnetic waves or sound with minimal loss of energy by restricting expansion to one dimension or more. A slotted wave guide is a wave guide that is used as an antenna in microwave radar application. A coaxial RF connector is an electrical connector designed to work at radio frequency in the multi band range. It is typically used with coaxial cables and are designed to maintain the shielding. The transmitter section consists of slotted waveguides antenna and magnetron. 


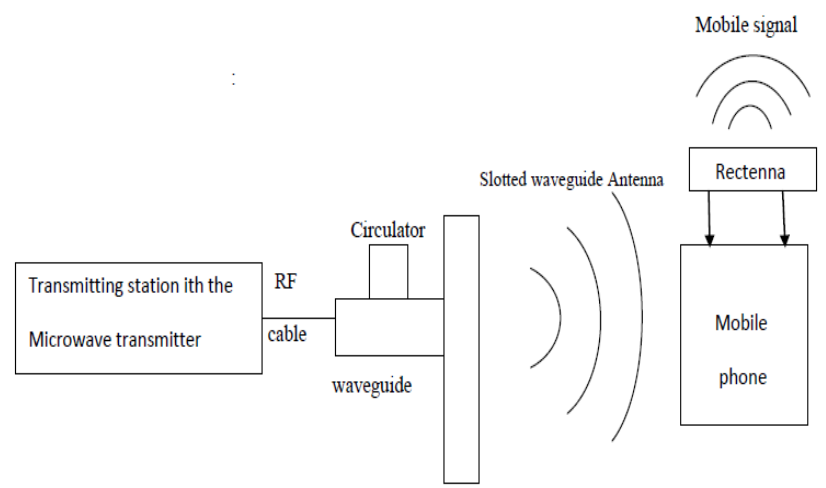

Fig. 1. Block Diagram of the Transmitter Section

Slotted waveguide antenna is an Omni directional antenna have high efficiency and gain of 13db .It has high power handling capacity.The magnetron is a vacuumtube oscillator that generates high power electromagnetic signal in the microwave frequency range. It is a microwave tube in which electron generated from a heated cathode are affected by magnetic and electric field to produce microwave radiation. It is used to produce high power output required in

\section{RECEIVER SECTION}

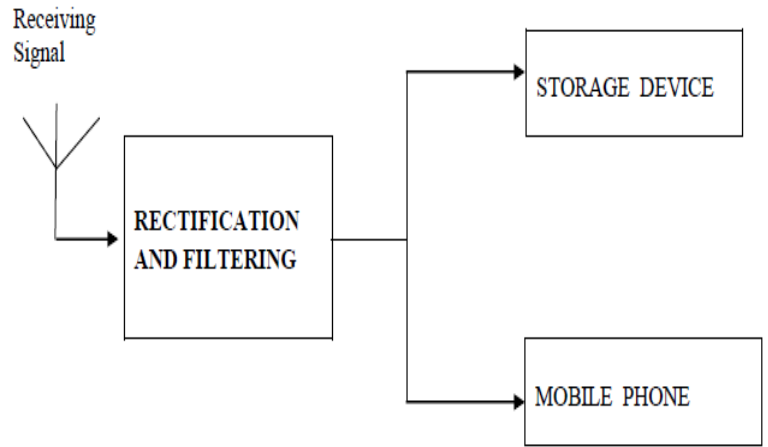

Fig. 2. Block Diagram of the Receiver Section

The basic addition to the mobile phone going to be the rectenna.it rectifies received microwaves into dc current. A rectenna comprises of a mesh of dipoles and diode for absorbing microwave energy from a transmitter and converting it into electric power. Its elements are usually arranged in a mesh pattern, giving it a distinct appearance from most antenna. Figure shows the received signal rectified to dc voltage,filtered and the voltage is stored in the storage device and the voltage is step downed to therequirement of the mobile phone necessity.

\section{BLOCK DIAGRAM}

When the microwave signal are transmitted from tower to mobile phone to connect the calls. The mobile phone consists of special device rectenna absorb the microwave signal then converted to dc voltage.

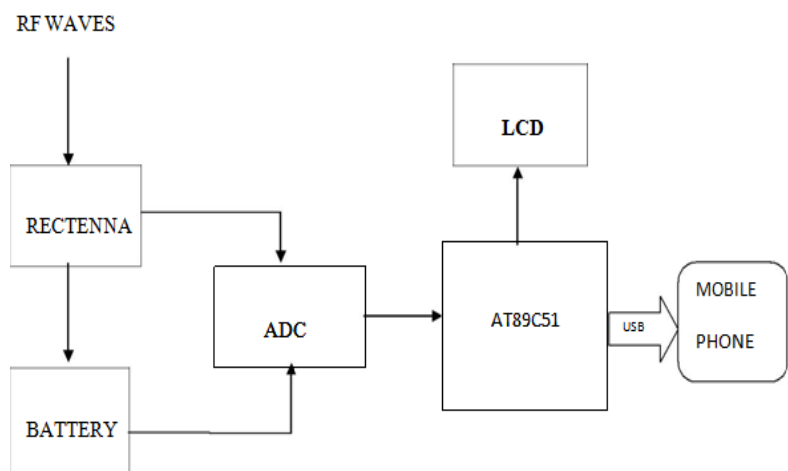

Fig. 3. Block Diagram of the Receiver Section 


\section{IJIREEICE \\ International Journal of Innovative Research in \\ Electrical, Electronics, Instrumentation and Control Engineering \\ ISO 3297:2007 Certified \\ Vol. 5, Issue 4, April 2017}

The rectenna operates at $2.45 \mathrm{ghz}$. The dc voltage are interfaced with ATMEL microcontroller. AT89C51 one of microcontroller is used for charging phones by microwaves. The LCD displays the output voltage from microcontroller with the help of rectenna. Then the output voltage is transferred to phone by USB cable.

\section{RECTENNA DESIGN}

A antenna which is used as trans receivers that is transmit and receives the signal. Along with that has its schottky diode is converts the RF wave into dc power. The diode works at $0.35 \mathrm{v}$ and it has lowest voltage drop and highest speed and therefore have the lowest power lost due to the conduction and switching. Impedance matching circuit is used to transfer maximum power and has the low power loss. The filter used in the circuit is low pass filter which absorbs the high frequency and allows low frequency to pass. A rectifying antenna or rectenna is one such means for recovering that energy. In order to demonstrate the capability of such a design a novel rectenna was developed for low power operation at the single industrial scientific medical(ISM) frequency of $2.45 \mathrm{GHz}$.

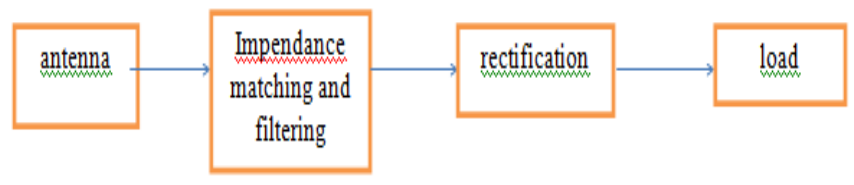

Fig. 4. Block Diagram of theRectanna Section

\section{PARAMETER OF RECTANNA DESIGN}
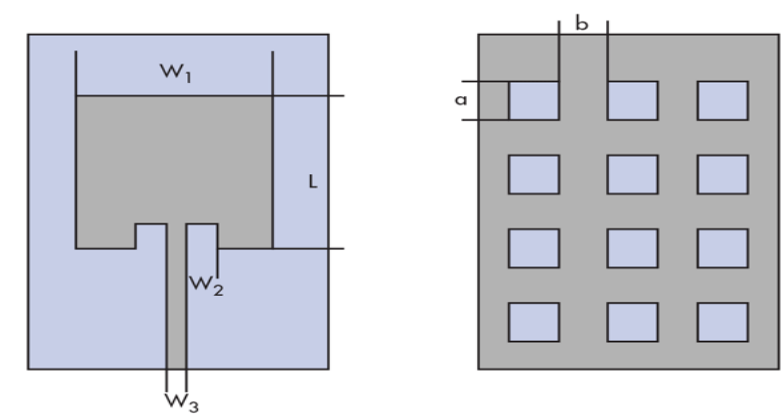

Fig. 5.Rectanna design

$\mathrm{W} 1=$ width of rectenna

$\mathrm{W} 2=$ width of slot in rectenna

W3=width of feed point in rectenna

A rectenna is an antenna with additional components, including a LPF and a rectifying circuit. The rectenna receives microwaves energy from the antenna. A schottky rectifier diode converts the received RF energy to DC power.The schottky diode also called as hot carrier diode, is a semiconductor diode formed by the junction of a semiconductor with the metal.it has a low forward voltage drop and a very fast switching action. When sufficient forward voltage is applied, a current flows in the forward direction. Thesilicon diode has typically forward voltage emf 600 to $700 \mathrm{mv}$. This lower forward voltage requirement allows higher switching speed and better system efficiency.The amount of power that can be transmitted is limited, and the amount of microwave power is reduced from the source through attenuation, mainly due to free space signal path loss. For use in portable device which in generally, usually have small dimension, an effective rectenna design should also have small dimension.

The rectenna consists of high efficient photonic band gap (PBG) structure, a micro strip low pass filter (LPF) with defected ground structure (DGS) circuitry and a schottky diode. To evaluate the rectenna, it was fabricated on a lowcost FR-4 printed circuit board (PCB) materials with a relative a dielectric constant in the $\mathrm{z}$ region at $2.45 \mathrm{GHz}$ and thickness of $1 \mathrm{~mm}$. rectenna achieves RF into dc conversion efficiency of $63 \%$ when processing received power of $18 \mathrm{dbm}$. In order to achieve higher order harmonic rejection of unwanted signals, the rectenna design employs a miniature microstrip LPF with DGS. The simple DGS is applicable on $50 \mathrm{ohm}$ feeder line to achievethe requires low pass filtering performance. The LPF with DGS can suppress and isolate second and third harmonics signal from the rectifying circuitry, allowing the rectenna to achieve its target high RF to DC conversion efficiency without processing unwanted signals. 


\section{IJIREEICE \\ International Journal of Innovative Research in \\ Electrical, Electronics, Instrumentation and Control Engineering \\ ISO 3297:2007 Certified \\ Vol. 5, Issue 4, April 2017}

\section{ADC}

An analog to digital convertor is a device that converts a physical quantity to digital number that represent the quantity amplitude.it receives the signals from rectenna and battery and it can take only the particular voltage from battery,adc 0808 is a successive approximation type with 8 channel (i.e ) it can directly access 8 single ended analog signal.

\section{BATTERY}

The battery is an electro chemical device that produce the voltage potential when placing metals of different acid solution. The open circuit voltage that develops as an part of an electro chemical reaction varies with the electrodes and the metals used. Applying the charge or discharge place the battery into closed circuit voltage condition. Charging raises the voltage and discharging lowers its simulating effects. The voltage behavior under the load and charge is governed by the current flow and the internal battery resistance. The low resistance produce low fluctuation under load or charger, the high resistance causes the voltage to swing excessively. Cold Temperature lowers the voltage and heat raises it.

\section{ATMEL MICROCONTROLLER}

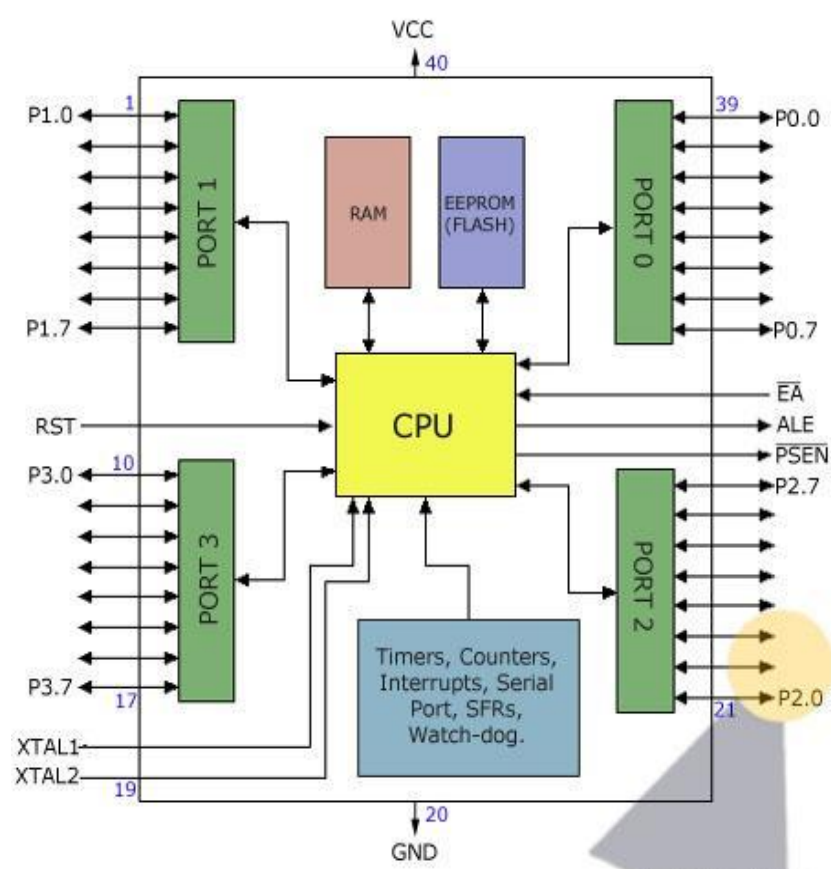

Fig.6.PIN Diagram of the ATMEL microcontroller

The AT89c51 is a low-power, high performance CMOS 8-bit microcontroller with 8k bytes of in-system programmable flash memory. The device is manufactured using Atmel high density non volatile memory technology and is compatible with the industry standard 80C51 instruction set and pin out.The on chip flash allows the program memory to be reprogrammed in system or by a conventional non volatile memory programmer.By combining a versatile 8 bit CPUwith in system programmable flash on monolithic chip,the ATMEL AT89c51 is a powerful microcontroller which provides a highly flexible and cost effective solution in many application. It provides the standard feature such as $4 \mathrm{~K}$ bytes of flask,128 bytes of RAM, 32 input output lines, two 16bit counter/ timers, 5 vector two level interrupt architecture, a full duplex serial pot, on chip oscillator and clock circuitry. It supports the power saving mode and idle mode.

\section{ADVANTAGE OF WIRELESS CHARGER}

Use of separate chargers is eliminated. Electricity is saved. The phone can be charged anywhere anytime. Lower risk of electrical shock because there are no exposed conductors, mobile phone is charged wirelessly. So time for charging is saved. Wastage of power is less and increased in efficiency. It is mainly depend upon the network coverage, so radiation problem may occur. The rate of charging may be minute range. 


\section{IJIREEICE \\ International Journal of Innovative Research in \\ Electrical, Electronics, Instrumentation and Control Engineering \\ ISO 3297:2007 Certified \\ Vol. 5, Issue 4, April 2017}

\section{CONCLUSION}

Thus the paper demonstrates a novel method of using the power of microwave to charge mobile phones without use of wired charger. It provides the great advantage to mobile phone user to carry their phones anywhere even if the place is devoid of facilities for charging. A novel utilization of the rectenna and a sensor in a cell telephone could give another measurement in the disclosure of cellular telephone. In this present day era where we favor the most productive devices to serve our reasons, not even a marginally veered off gadget is satisfactory. The exceedingly fulfilled wireless sensor made by the precisely topnotch makers in the business benefit our needs the most ideal way and turn out to be very powerful apparatuses to battle security rupture.

\section{REFERENCES}

[1] Wireless power transfer for mobile phone charging device.Olvitz.C,Vinko. D,Svedek.T.MIPRO, 2012 proceddings of 35 th.intersation convention.

[2] A.Kurs,A.Karalis,R.Moffat,J.D.Joannopoulos ,P.Fisher and M.Soljacie “wireless power transfer via srongly coupled magnetic reasonances,"SCIENCE vol.317,july 2001.

[3] B.Leanaerts and R.Puers "Inductive powering of freely moving system" sensors and acutators vol.A123 124.pp.522-530,2006.

[4] Liou,chong-yi;chi,Jungkuo,Ming lung la,Shau-gang mao Microwave symposium digest (MTT),2012 -IEEE MTT-Sinternational.

[5] A.P.Sample,D.A.Meyer and L.R.Smith "Analysis experimental results and range adaption of magnetically coupled reasonators for wireless power transfer,"IEEE“"

[6] I.Garnica,I.Casunova and I.Lin "High off mid range wireless power system in 2011 IEEE MTT-S-information microwave workshop series on innovative wireless power transmission technologies system and applications.(IMWS), Kyoto,may 2011 pp.73-76.

[7] IEEE microwave theory \& techniques, Bill Brown's distinguished career. Mtt.org. retrieved 4 June 2009.

[8] Woy Key, Elizabeth (o2-12-2010). "wireless charging goes mainstream".forbes.com.

[9] Wireless electricity specification meaning completion".PC world . 18 August 2009. Retrieved 21 August 2009.

[10] Energizer inductive charger for Wii preview: .IGN.com.2009-04-28.

[11] Wireless power transfer for mobile phone charging device.Olvitz.C,Vinko. D,Svedek.T.MIPRO, 2012 proceddings of 35 th.intersation convention.

[12] Kurs,A.Karalis, R. Moffat, J.D. Joannopoulos ,P.Fisher and M.Soljacie "wireless power transfer via srongly coupled magnetic reasonances,"SCIENCE vol.317,july 2001.S

[13] B.Leanaerts and R.Puers "Inductive powering of freely moving system" sensors and acutators vol.A123 124.pp.522-530,2006.

[14]Liou,chong-yi;chi,Jungkuo,Ming lung la,Shau-gang mao Microwave symposium digest (MTT),2012 -IEEE MTT-Sinternational. 